\title{
Search of the Control Algorithm for a High-Resolution Automated Resistor
}

\author{
Panhui Chen, Lifeng Guo \& Chuanxi Jin \\ Naval University of Engineering, Wuhan 430033, China
}

\begin{abstract}
This article describes an automated resistor that is based on a resistor network and the control algorithm of successive approximations. The resistor network, consists of relays and precise resistors, has a structure of reducing the effect of relay' contacts resistance. The nonlinear error of the network without the mismatch can be calibrated by the successive approximation algorithm. The accuracy test shows that, the output resistance error less than $\pm 2 \mathrm{~m} \Omega$, with the range of 71 to $78 \Omega$ and the step of $1 \mathrm{~m} \Omega$.
\end{abstract}

KEYWORD: automated resistor; control algorithm; successive approximation; nonlinear

\section{INTRODUCTION}

The resistance-thermometry instruments with the sensors of pt100 are generally used in the highprecision temperature measurements (H Rosemary Taylor et al, 1983) (P C F Wolfendale, 1969). As the nonlinearity relate to the measured temperature and resistance of pt100, varies from calibration methods, such as the analog positive feedback control, seeking the nonlinearity parameter table (LI Tianhui et al, 2011), least squares method (WU Dajun et al, 2011), Newton method and analytical method, are used in the pt100 based instruments to improve the accuracy of the temperature measurement. Variable resistors, such as resistance boxes, electronic synthesized resistors and automated resistors based on networks which insist of relays and precision resistors, are usually used to the calibration effect evaluation of the instrument.

In this article, a resistor network with a resolution of 0.001 is described. To deal with the nonideal characteristics of the precision resistors and relays, such as the initial resistance error and the random of the relays' contact resistance, a nonlinearity control algorithm is also developed in this article.

\section{RESISTOR NETWORK}

A network circuit is shown in figure 1 which consists of resistors and relays.

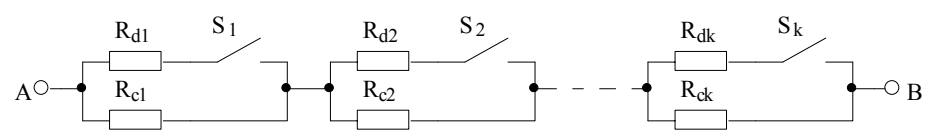

Fig. 1: The network circuit

The network can be seemed ask elements in serial and the terminals of A and B is the output terminals. Every element consists of a main resistor $R_{c x}$, a slave resistor $R_{d x}$ and a relay. As the relay is open, the resistance of the element equals to $R_{c x}$, otherwise, the resistance of element $R_{x}$ equals to the relay' contact resistor $R_{c t}$ series with the slave resistor $R_{d x}$ and then parallels with the main resistor $R_{c x}$.

The $R_{x}$ can be calculated as:

$R_{x}=\frac{R_{\mathrm{cx}}\left(R_{\mathrm{c} x}+R_{c t}\right)}{R_{\mathrm{c} x}+R_{\mathrm{c} x}+R_{c t}}$

As described in Figure 1, the network resistance $R$ equals to the sum of every element resistance.

$R_{\text {out }}=\sum_{x=1}^{x=k} R_{\mathrm{x}}$

The $R$ reach to the maxim value $R_{\max }$, as all of the relays is open, and $R$ reach to the minim values $R_{\min }$ when all of the relays closure. Hence, the resistor range of the resistor network equals $\left[R_{\min }, R_{\max }\right]$. 
$R_{\max }=\sum_{x=1}^{x=k} R_{c x}$

$$
R_{\min }=\sum_{x=1}^{x=k} R_{\mathrm{x}}
$$

The $R$ value change after any relay's state changed. $\mathrm{K}$ relays can realize $2^{\mathrm{k}}$ states. As a k dimensions binary variable $P=\left[P_{1}, P_{2}, \ldots P_{K}\right]$ is assumed, and the $\mathrm{k}$ resistance changes is defined as $\Delta R=\left[\Delta R_{1}, \Delta R_{2}, \ldots \Delta R_{K}\right]^{T}$ the network resistance $R$ can be calculated as:

$$
\begin{aligned}
& R=\sum_{x=1}^{x=k} R_{c x}-\sum_{x=1}^{x=k} \Delta R_{x} \\
& R=R_{\min }+P \cdot \Delta R
\end{aligned}
$$

\section{ALGORITHM DESIGN}

According to the network resistance calculation in (10), the mathematic module of the network is similar to a linear neural network, which is shown in figure 2.

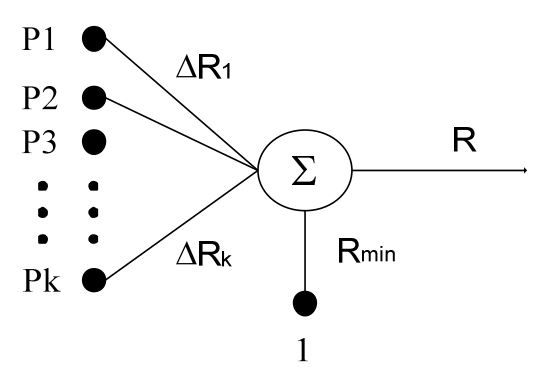

Figure 2 The mathematic module of network

The $P_{1} \sim P_{k}$, can be only set as " 0 " or " 1 ", are the digital inputs, the $\Delta R_{1} \sim \Delta R_{k}$ are the weights of the branches, and $R_{\min }$ is the fixed offset. If we define a k-bit value $D$ as:

$$
D=\sum_{i=0}^{i=k} 2^{i} P_{i}
$$

Only, the relationship between network resistance $R$ and input value $D$ is linear, as $\Delta R_{x}$ is a binary array, which value is $\Delta R_{x}=2^{x} \times \Delta R_{1}$. In this situation, the control digital bits can be simple calculated as $D=\left(R_{o}-R_{\min }\right) / \Delta R_{1}$, when an output resistance requirement $R_{o}$ is given. However, the weight $\Delta R_{x}$ is depending on the actual resistance of $R_{c x}, R_{d x}, R_{c t}$, and even the PCB line. As a random initial error exists on every precision resistor, the actual weights are too hard to be fixed at the ideal values. Therefore, another precise and fast algorithm is needed to be developed.

Refer to the concept of the algorithm for a successive approximation register (SAR) analog-to-digital converters [10], the calculation of control bits $P_{k}$ can be separated out $\mathrm{k}+1$ steps. At the beginning, the weights should be arranged to a descending series $\left(\Delta R_{1}>\Delta R_{2} \ldots>\Delta R_{k}\right)$. Then, the successive approximation algorithm set the control bits which are mostly close to the ideal values.

\section{CIRCUIT TEST}

Table 1: Actual parameters

\begin{tabular}{llll}
\hline Number & Name & $\begin{array}{l}\text { Average values } \\
(\Omega)\end{array}$ & Variances \\
\hline 1 & $\Delta R_{15}$ & 0.001059 & $3.25 \mathrm{E}-07$ \\
2 & $\Delta R_{14}$ & 0.001999 & $2.13 \mathrm{E}-07$ \\
3 & $\Delta R_{13}$ & 0.003067 & $2.71 \mathrm{E}-07$ \\
4 & $\Delta R_{12}$ & 0.004045 & $2.60 \mathrm{E}-07$ \\
5 & $\Delta R_{11}$ & 0.009989 & $2.05 \mathrm{E}-7$ \\
6 & $\Delta R_{10}$ & 0.019695 & $3 \mathrm{E}-7$ \\
7 & $\Delta R_{9}$ & 0.029294 & $2.46 \mathrm{E}-7$ \\
8 & $\Delta R_{8}$ & 0.039627 & $2.18 \mathrm{E}-7$ \\
9 & $\Delta R_{7}$ & 0.098829 & $2.88 \mathrm{E}-7$ \\
10 & $\Delta R_{6}$ & 0.202734 & $1.96 \mathrm{E}-7$ \\
11 & $\Delta R_{5}$ & 0.297388 & $2.28 \mathrm{E}-7$ \\
12 & $\Delta R_{4}$ & 0.399648 & $2.29 \mathrm{E}-7$ \\
13 & $\Delta R_{3}$ & 0.993135 & $2.70 \mathrm{E}-7$ \\
14 & $\Delta R_{2}$ & 1.969162 & $2.35 \mathrm{E}-7$ \\
15 & $\Delta R_{1}$ & 3.037341 & $2.37 \mathrm{E}-7$ \\
16 & $R_{\min }$ & 70.95399 & $1.31 \mathrm{E}-7$ \\
\hline
\end{tabular}

As discussed in figure 4, the accuracy of the successive approximation algorithm depends on the resistance of the weights. The mathematic value in table 1 is unauthentic, and the actual weights should be measured after the network hardware assembled. An automatic weight measure system, based on virtual instrument, was developed. It consists of a computer, the automated resistor network which connects to the computer via USB, and an $81 / 2$ digital multimeter HP3458A, which connects to the computer via GPIB. The software in the computer is based on LabVIEW. 240 sets of measure data was capture by the weight measure system. Depend on 
the data, the average values and the variances of the weights was calculated, which is shown in table 1 .

According to the actual parameter, the maxim network' mismatch error equals 0.00106 . The data of the weights measurement presents random properties. As the variances approximate $10^{-7}$, the average of the measurement data can be authentic to instead the actual weights.

After the actual weights measurement, the control software, based on the successive approximation algorithm, was developed. The control software was suited to the actual resistor network with the calculation parameter import.

As all the control bits set to " 1 ", the resistance of the network will reach to $R_{\max }$. Otherwise, the resistance will reach to $R_{\min }$, when all the control bits set to "0". The $81 / 2$ multimeter HP3458A was used to measure the resistance of the two situations, and the $R_{\max }$ equals to $70.9954 \Omega$, the $R_{\min }$ equals to $78.0609 \Omega$. Hence, the resistance network value ranges from $70.9954 \Omega$ to $78.0609 \Omega$.

For the sake of simplicity, a serial resistance values range from $71 \Omega$ to $78 \Omega$ with a step of $0.1 \Omega$ was chooses to be the test points to estimate the accuracy of the network. Also, an automated system, similar to the weights measurement, was used for a 60 time tests of every test point, and the errors is shown in figure 3 .

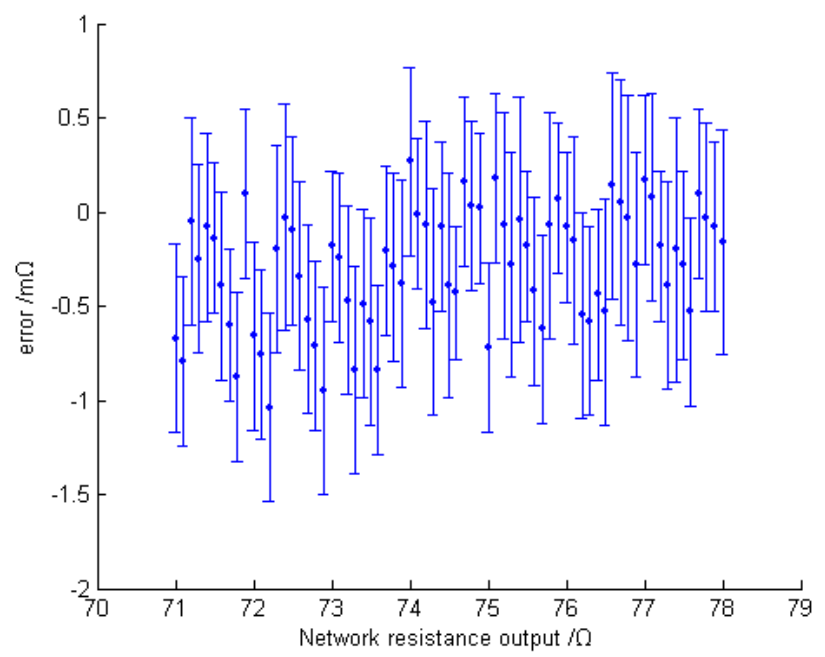

Figure 3 Resistance out error test result

\section{CONCLUSION}

We realized an automated resistor for testing and calibrating the resistance-thermometry instruments. By using the slave resistor, the random effect of relays' contact resistance is reduced. Therefore, the stability of the network resistance is improved. As the initial error of the precise resistors and the effect of the relays and the PCB line in an actual circuit, mismatch and linear error is the key fact to influence the output accuracy. According to the analysis of the mismatch condition, a network with the weights changed can realize a network without mismatch error. The control algorithm of successive approximation is preferably used to eliminate the nonlinear error, with the actual weights measured accurately.

Accuracy test of the output resistance shows that the present resistor network can provide a stable and precise resistance output. As the random error of the multimeter, the output resistance value varies at each measurement. But, the total error of the output resistance is less than $\pm 2 \mathrm{~m} \Omega$.

\section{REFERENCES}

H Rosemary Taylor, Hector a Navarro: A microcomputerbased instrument for applications in platinum resistance thermometry, J. Phys. E: Sci. Instrum, Vol. 16(1983), p. 1100 .

LI Tianhui, WEI Shan, YU Qian, HONG Xingen, LU Chao: Design of High-Temperature Thermometer Based on Pt100 Using Look-up Table, Electronic Sci, \& Tech., Vol. 24(2011) No.10, p.52.

P C F Wolfendale: A precise automatic a.c. potentiometer for low temperature resistance thermometry, J. Phys. E: Sci. Instrum, Vol. 2(1969), p. 659.

WU Dajun, Yang Shanshan, Qiu chengjun: InductanceFrequency Converting Magnetron System for Measurement, Journal of Anhui Agri. Sci., Vol. 39(2011) No.20, p.11989. 